Supporting Information

\title{
Ladder-type Dye with Large Transition Dipole Moment for Solvatochromism and Microphase Visualization
}

Jian Cao, Qi-Ming Liu, Si-Jie Bai, Hua-Chun Wang, Xiancheng Ren and Yun-Xiang Xu*

College of Polymer Science \& Engineering, State Key Laboratory of Polymer Materials Engineering, Sichuan University, Chengdu 610065, China.

*E-mail: yxxu@scu.edu.cn 
Scheme S1. Schematic representation and examples of (a) TICT and (b) PICT model.

(a)
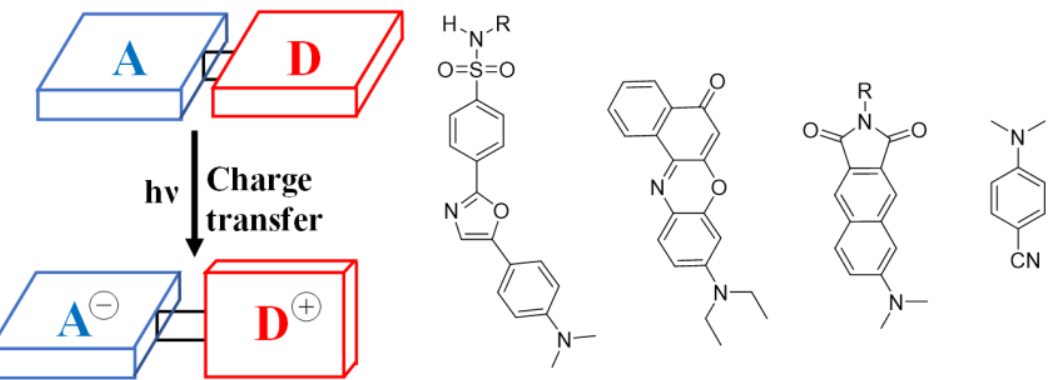

(b)

TICT

Dapoxyl

Nile Red

6DMN DMABN
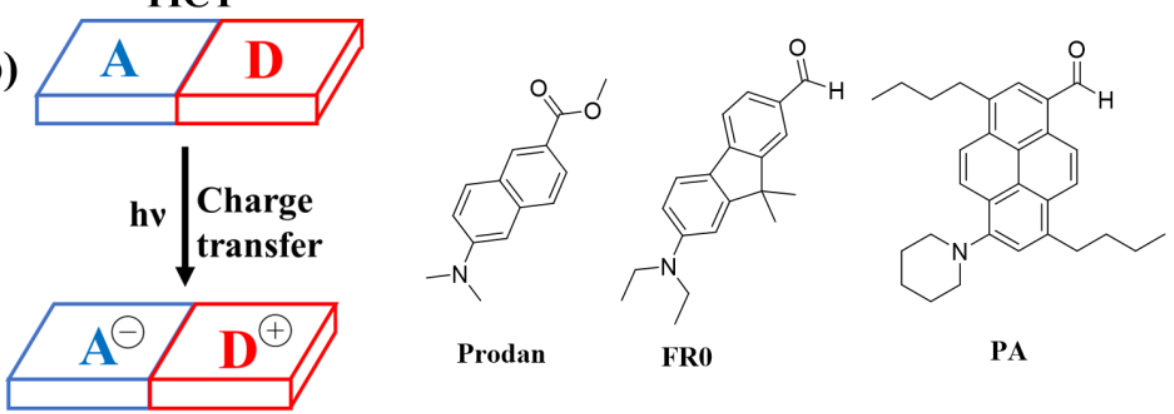

PA

PICT

Scheme S2. Synthetic route of ISOAM-H.
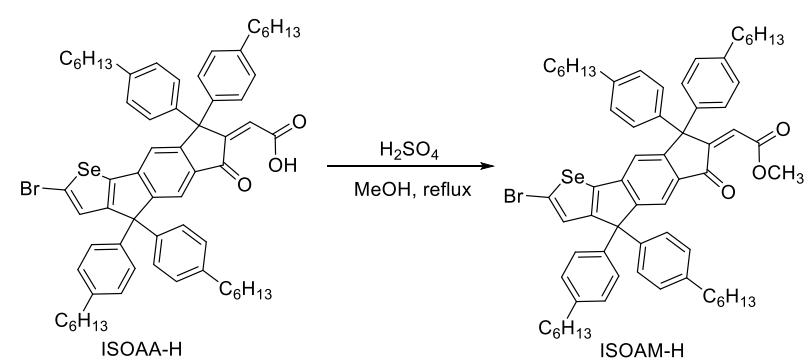

\section{Synthesis of ISOAM-H}

ISOAA-H (86 mg, $0.0820 \mathrm{mmol})$ was added into methanol $(10 \mathrm{~mL})$. Then, 3 drops of concentrated sulfuric acid was added carefully. After refluxing for $4 \mathrm{~h}$, the precipitation was filtered and purified by silica gel chromatography (petroleum ether/ethyl acetate $=30: 1)$ to afford a yellow solid $(34 \mathrm{mg}, 39 \%)$. ${ }^{1} \mathrm{H}$ NMR (400 MHz, $\left.\mathrm{CDCl}_{3}, \delta\right) 7.77$ (s, 1H), 7.29 (s, 1H), 7.25 (s, 1H), 7.13 (dd, $\left.J=18.6,8.3 \mathrm{~Hz}, 8 \mathrm{H}\right)$, $7.04(\mathrm{~s}, 8 \mathrm{H}), 6.03(\mathrm{~s}, 1 \mathrm{H}), 3.84(\mathrm{~s}, 3 \mathrm{H}), 2.60-2.51(\mathrm{~m}, 8 \mathrm{H}), 1.63-1.52(\mathrm{~m}, 8 \mathrm{H}), 1.31-1.26(\mathrm{~m}, 24 \mathrm{H})$, $0.89-0.86$ (m, 12H). ${ }^{13} \mathrm{C}$ NMR (100 MHz, $\left.\mathrm{CDCl}_{3}, \delta\right)$ 190.87, 167.74, 160.06, 158.17, 152.84, 151.82, 147.27, 143.80, 142.40, 142.22, 142.05, 140.35, 134.04, 129.53, 128.69, 128.66, 127.78, 126.60, 121.57, $121.01,117.43,64.62,59.66,52.43,35.67,35.64,31.85,31.44,31.42,29.27,29.22,22.74,14.24$.

\section{Preparation of ISOAA-H-doped polymer films}

Polymer sample was dissolved in good solvents (PIP, SBS, PMMA and PS in toluene; PEG in chloroform) to prepared a solution with concentration of $50 \mathrm{mg} / \mathrm{mL}$, respectively. Then polymer blend 
solutions were prepared by mixing equal volumes of two different polymer solutions. And ISOAA-H was added to the mixture with a mass fraction of $1 \%$ of polymers. Then the solution was stirred for $2 \mathrm{~h}$. The ISOAA-H-doped polymer blend films were prepared by spin-coating the mixture solutions onto quartz glass $\left(1 \mathrm{~cm}^{2}\right)$ at $600 \mathrm{rpm}$ for $45 \mathrm{~s}$. Then, the obtained films were dried in air.

\section{The Lippert-Mataga equation ${ }^{1-3}$}

$$
\begin{gathered}
\Delta v=v_{\mathrm{A}}-v_{\mathrm{F}}=\frac{2 \Delta \mu^{2}}{h c a_{0}^{3}} \Delta f+\text { const } \\
\Delta \mu=\mu_{\mathrm{e}}-\mu_{\mathrm{g}} \\
\Delta f=\frac{\varepsilon-1}{2 \varepsilon+1}-\frac{n^{2}-1}{2 n^{2}+1} \\
a_{0}=\left(\frac{3 M}{4 N \pi d}\right)^{\frac{1}{3}}
\end{gathered}
$$

where $\Delta v$ is the Stokes shift (in $\mathrm{cm}^{-1}$ ) between the absorption and fluorescence emission peak, $h$ is the Planck constant, $c$ is the velocity of light in vacuum, $\Delta \mu$ is the transition dipole moments between the ground $\left(\mu_{\mathrm{g}}\right)$ and excited $\left(\mu_{\mathrm{e}}\right)$ states, $\Delta f$ is the orientation polarizability of the solvents expressed by the dielectric constant $(\varepsilon)$ and refractive index (n) of the solvents and $a_{0}$ is the Onsager cavity radius, which can be derived from the Avogadro number $N$, molecular weight $M$, and density $d\left(d=1.0 \mathrm{~g} / \mathrm{cm}^{3}\right)$. The Onsager cavity radius $\left(a_{0}\right)$ of ISOAA-H was calculated to be $7.467 \AA$ according to the equation (4).
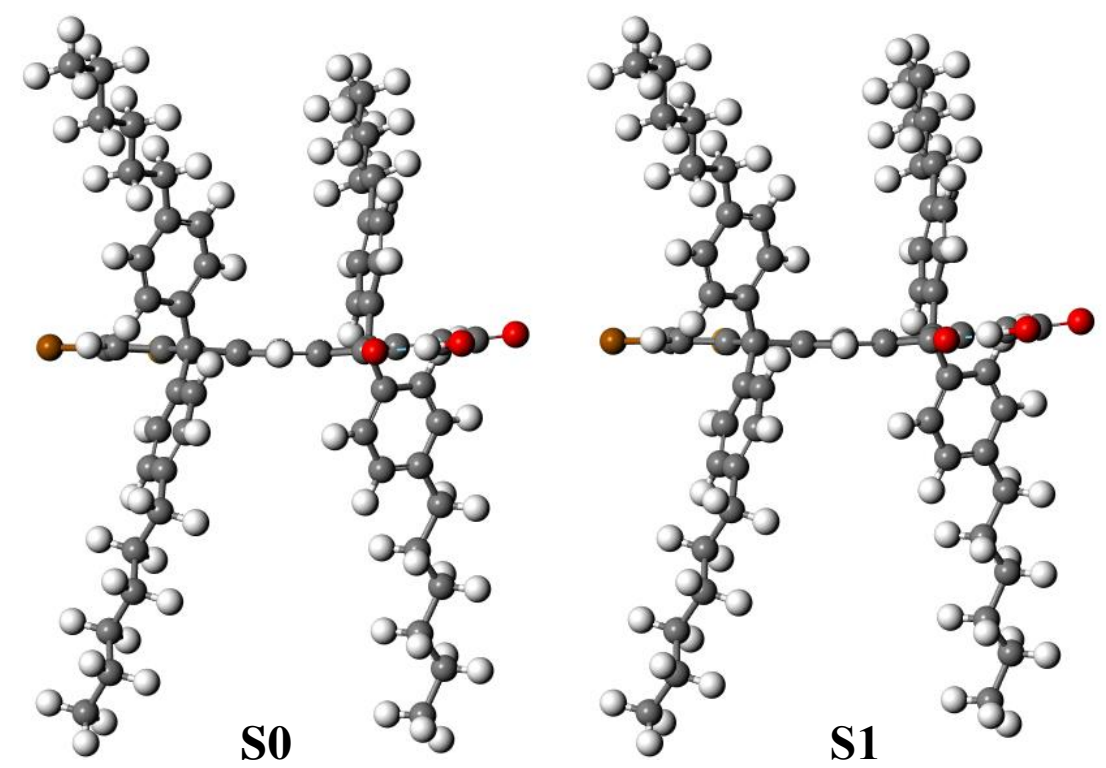

Figure S1. Side view of the geometry-optimized structures of ISOAA-H in ground state $\left(\mathrm{S}_{0}\right)$ and excited state $\left(\mathrm{S}_{1}\right)$. 

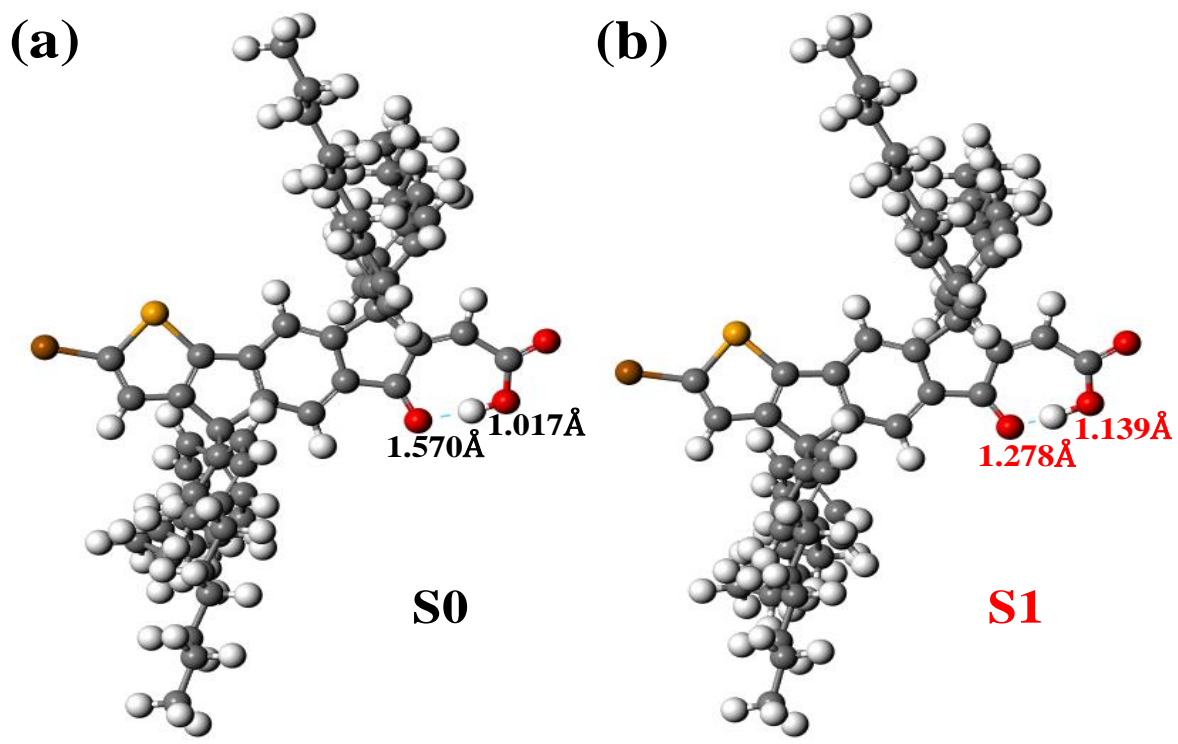

Figure S2. Optimized geometries of ISOAA-H in (a) ground state and (b) excited state.

Table S1. The Stokes shift of ISOAA-H in various solvents with a range of $\Delta f$ (orientation polarizability) values.

\begin{tabular}{ccccc}
\hline Solvents & $\varepsilon$ & $n$ & $\Delta f^{\mathrm{a}}$ & Stokes shift $\left(\mathrm{cm}^{-1}\right)$ \\
\hline Toluene & 2.38 & 1.494 & 0.014 & 2953 \\
Chloroform & 4.9 & 1.446 & 0.151 & 3680 \\
Ethyl acetate & 6.02 & 1.372 & 0.2 & 4579 \\
Tetrahydrofuran & 7.58 & 1.405 & 0.21 & 4372 \\
Dichloromethane & 8.93 & 1.424 & 0.217 & 4244 \\
Acetone & 20.7 & 1.359 & 0.284 & 5534 \\
Acetonitrile & 37.5 & 1.344 & 0.305 & 5751 \\
\hline
\end{tabular}

${ }^{\text {a }}$ Calculated from equation (3). 
Scheme S3. Molecular structure and proposed photophysical process of solvatochromic dye ISOAA-H in this work. ICT* represents intramolecular charge transfer state; IPCT* represents intramolecular proton shift enhanced charge transfer state.
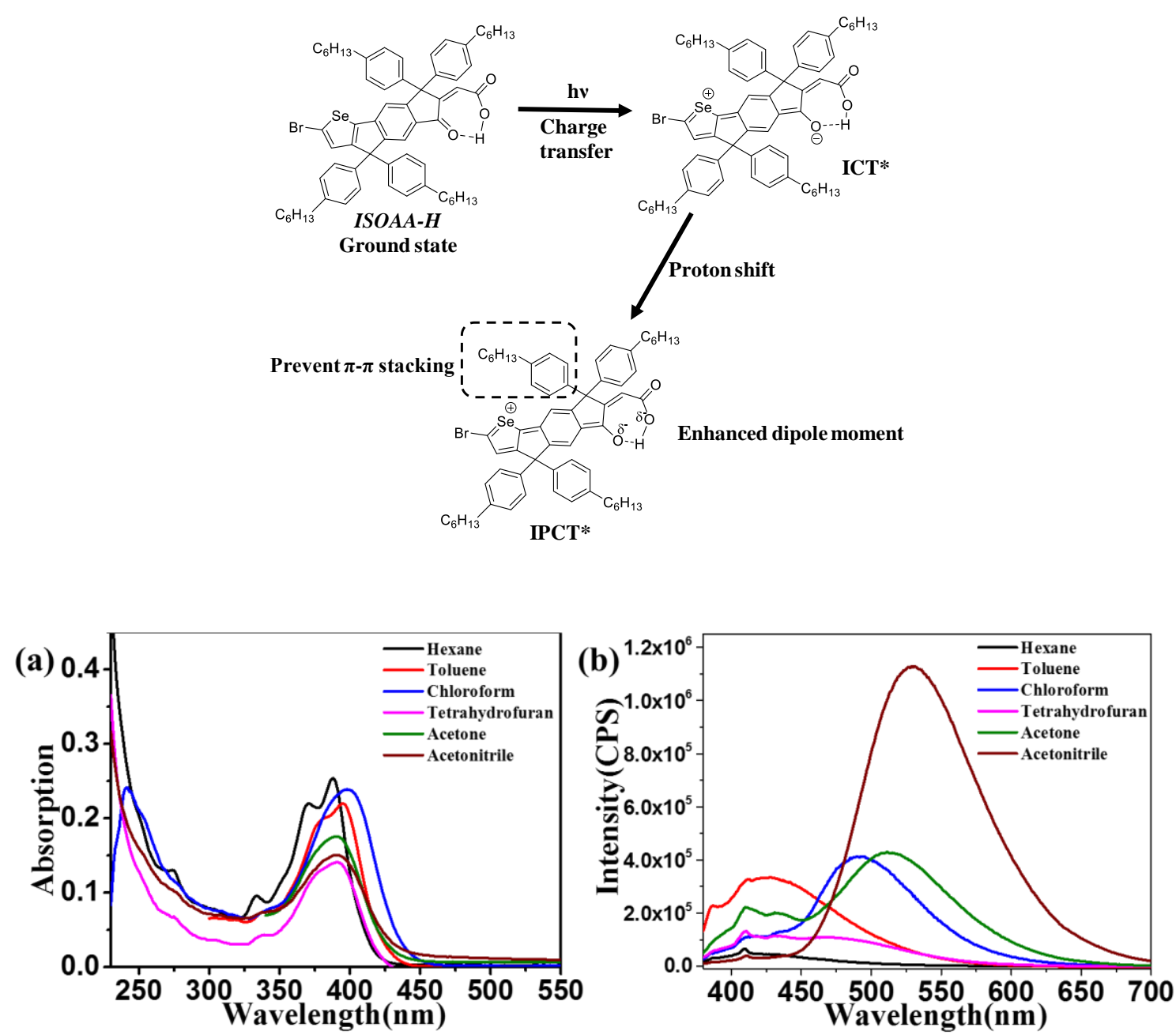

Figure S3. (a) UV-Vis absorption and (b) PL spectra of ISOAM-H in various solvents. Excitation wavelength: 365 $\mathrm{nm}, \mathrm{c}=10^{-5} \mathrm{M}$.

Table S2. Photophysical properties of ISOAM-H in various solvents.

\begin{tabular}{cccc}
\hline Solvents & $\lambda_{\text {abs }}{ }^{\mathrm{a}}(\mathrm{nm})$ & $\begin{array}{c}\varepsilon \\
\left(10^{4} \mathrm{M}^{-1} \mathrm{~cm}^{-1}\right)\end{array}$ & $\lambda_{\mathrm{em}}{ }^{\mathrm{b}}(\mathrm{nm})$ \\
\hline Hexane & 388 & 2.54 & - \\
Toluene & 395 & 2.20 & 428 \\
Chloroform & 398 & 2.39 & 469 \\
Tetrahydrofuran & 391 & 1.41 & 514 \\
Acetone & 390 & 1.76 & 529 \\
Acetonitrile & 391 & 1.51 & 492 \\
\hline
\end{tabular}

${ }^{\mathrm{a}}$ Longest wavelength absorption maximum. ${ }^{\mathrm{b}} \mathrm{CT}$ emission maximum $\left(\lambda_{\mathrm{ex}}=365 \mathrm{~nm}\right)$. 


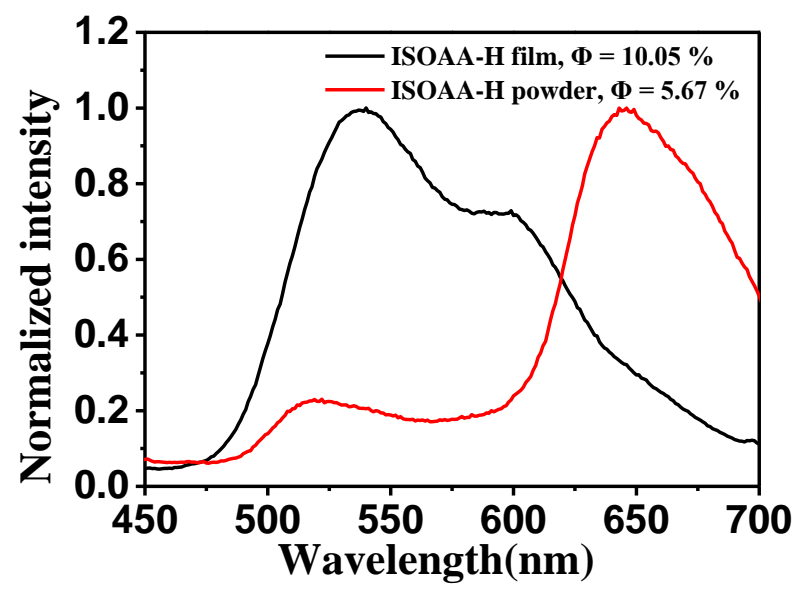

Figure S4. Normalized photoluminescence spectra of ISOAA-H as film and powder. Excitation wavelength: $365 \mathrm{~nm}$. (The film was prepared by spin-coating a chloroform solution on quartz glass)

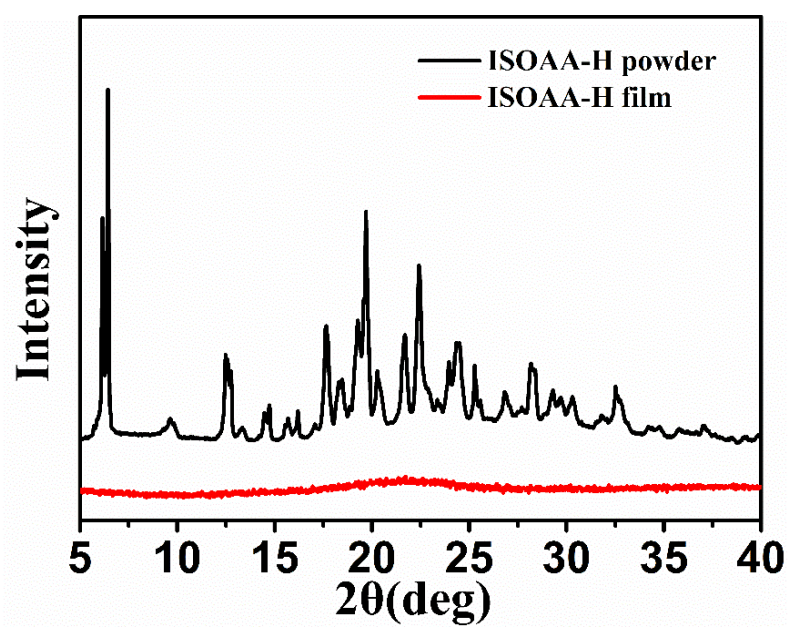

Figure S5. XRD patterns of ISOAA-H as film and powder. (The film was prepared by spin-coating a chloroform solution on quartz glass)
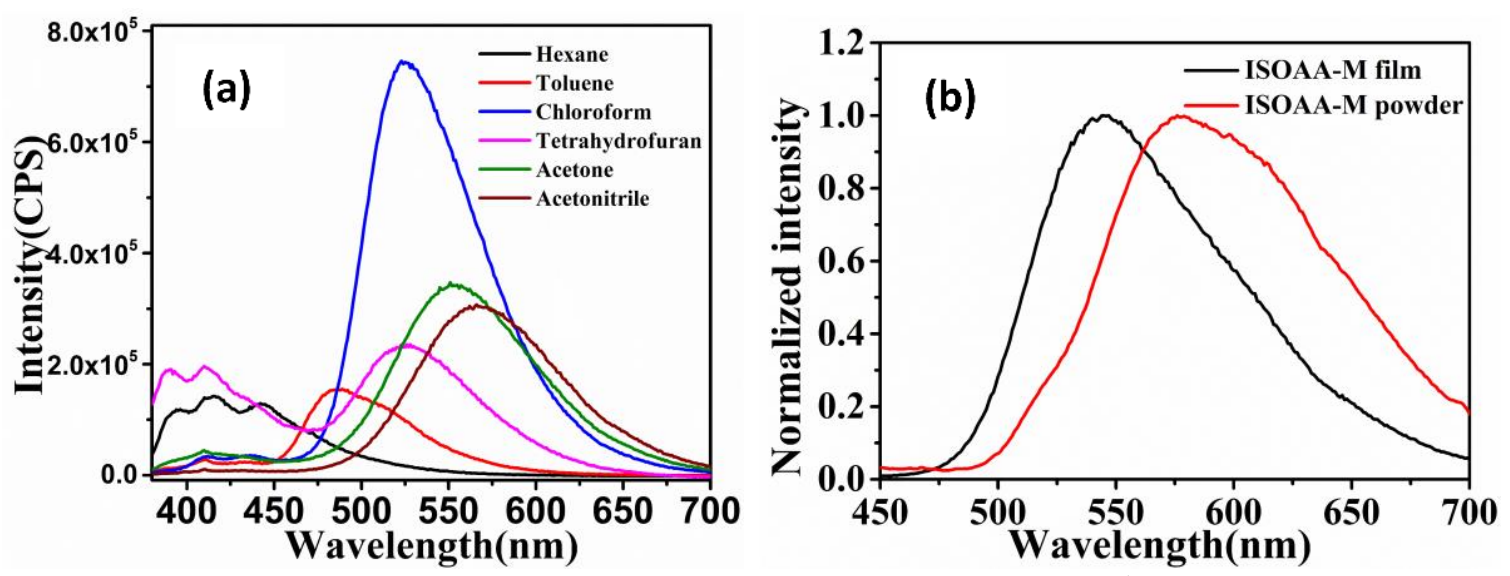

Figure S6. Photoluminescence spectra of ISOAA-M (a) in various solvents $\left(\mathrm{c}=10^{-5} \mathrm{M}\right)$ and (b) as film and powder. Excitation wavelength: $365 \mathrm{~nm}$. (The film was prepared by spin-coating a chloroform solution on quartz glass) 

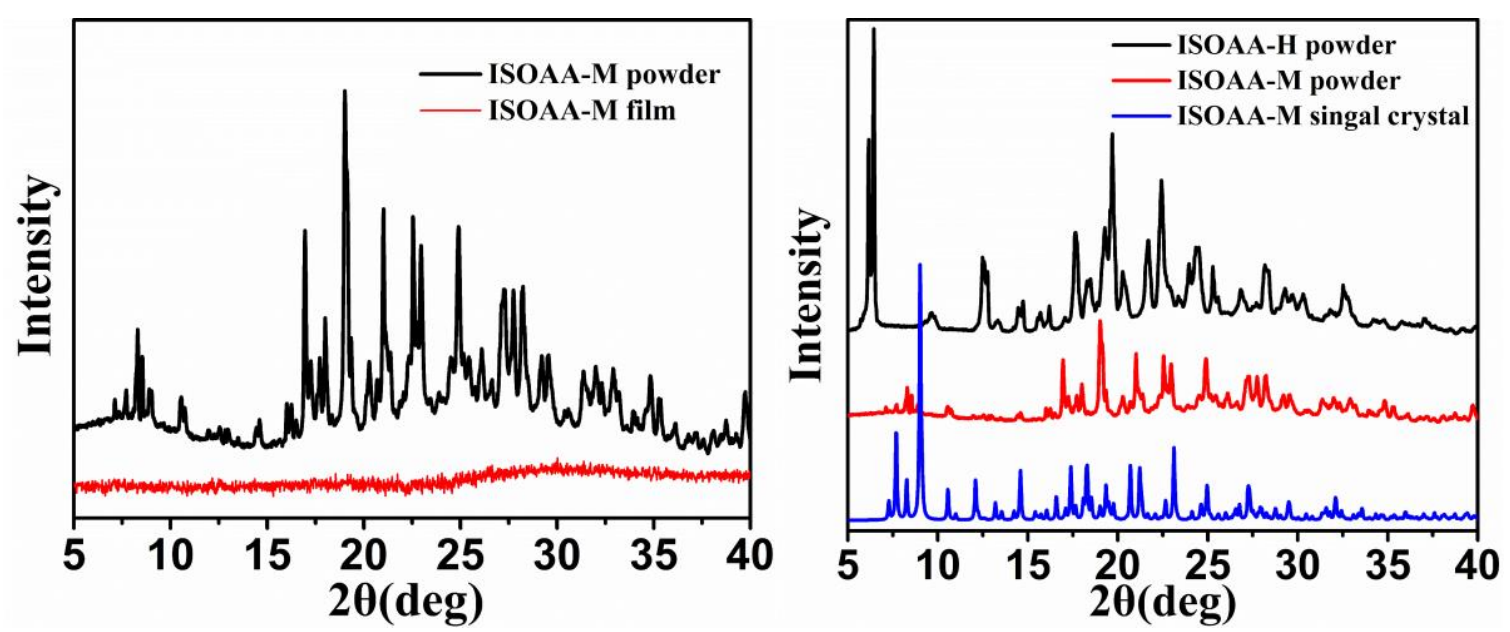

Figure S7. XRD patterns of ISOAA-H and ISOAA-M as films and powder. (The film was prepared by spin-coating a chloroform solution on quartz glass)
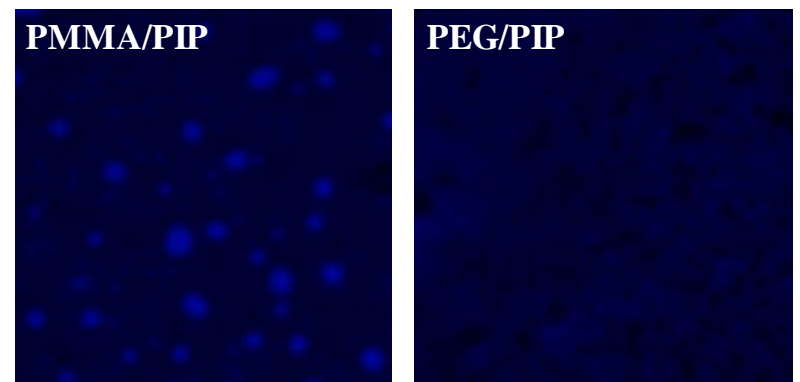

Figure S8. Fluorescent images of $1.0 \mathrm{wt} \%$ ISOAA-H doped polymer blend films of PMMA/PIP (50/50 wt/wt) and PEG/PIP (50/50 wt/wt). $1500 \times 1500 \mu \mathrm{m}^{2}$ for PMMA/PIP blend and $2000 \times 2000 \mu \mathrm{m}^{2}$ for PEG/PIP blend.

Table S3. The Cartesian coordinates $(\AA)$ of the optimized geometries for ISOAA-H in ground state.

\begin{tabular}{ccccc}
\hline & atom & $\mathrm{X}$ & $\mathrm{Y}$ & $\mathrm{Z}$ \\
\hline 1 & $\mathrm{C}$ & -6.208303 & -5.384085 & -0.188601 \\
2 & $\mathrm{C}$ & -6.181124 & -6.560440 & -1.003082 \\
3 & $\mathrm{C}$ & -5.726703 & -6.493098 & -2.304019 \\
4 & $\mathrm{C}$ & -5.260502 & -5.248027 & -2.771775 \\
5 & $\mathrm{C}$ & -5.265190 & -4.091953 & -1.970262 \\
6 & $\mathrm{C}$ & -5.756869 & -4.143568 & -0.666143 \\
7 & $\mathrm{C}$ & -4.596027 & -2.908895 & -2.683110 \\
8 & $\mathrm{C}$ & -4.194718 & -3.547701 & -4.040040 \\
9 & $\mathrm{C}$ & -4.637457 & -4.966670 & -4.059303 \\
10 & $\mathrm{O}$ & -4.488559 & -5.788615 & -4.992003 \\
11 & $\mathrm{O}$ & -3.198095 & -4.498886 & -6.820285 \\
12 & $\mathrm{C}$ & -3.034859 & -3.248501 & -6.366079 \\
13 & $\mathrm{C}$ & -3.543747 & -2.864051 & -5.011962 \\
14 & $\mathrm{C}$ & -6.706805 & -5.792116 & 1.102754 \\
15 & $\mathrm{C}$ & -6.979557 & -7.149180 & -135853 \\
16 & $\mathrm{C}$ & -6.653446 & -7.797506 & 2.364184 \\
17 & $\mathrm{C}$ & -7.475904 & -7.652287 & 3.321016 \\
18 & $\mathrm{C}$ & -7.622163 & -6.675504 &
\end{tabular}




\begin{tabular}{|c|c|c|c|c|}
\hline 19 & $\mathrm{Se}$ & -7.090669 & -4.947676 & 2.735245 \\
\hline 20 & $\mathrm{C}$ & -5.503681 & -8.810700 & -0.009303 \\
\hline 21 & $\mathrm{C}$ & -4.166855 & -8.375769 & 0.012423 \\
\hline 22 & $\mathrm{C}$ & -3.125285 & -9.278299 & 0.210507 \\
\hline 23 & $\mathrm{C}$ & -3.361853 & -10.652168 & 0.392371 \\
\hline 24 & $\mathrm{C}$ & -4.696681 & -11.077410 & 0.407135 \\
\hline 25 & $\mathrm{C}$ & -5.750672 & -10.170744 & 0.217163 \\
\hline 26 & $\mathrm{Br}$ & -8.309957 & -6.917117 & 5.070172 \\
\hline 27 & $\mathrm{O}$ & -2.457469 & -2.389441 & -7.029283 \\
\hline 28 & $\mathrm{C}$ & -3.379737 & -2.485498 & -1.831319 \\
\hline 29 & $\mathrm{C}$ & -5.547208 & -1.730756 & -2.963286 \\
\hline 30 & $\mathrm{C}$ & -3.535464 & -1.560763 & -0.787753 \\
\hline 31 & $\mathrm{C}$ & -2.456999 & -1.199456 & 0.026166 \\
\hline 32 & $\mathrm{C}$ & -1.182011 & -1.748596 & -0.162772 \\
\hline 33 & $\mathrm{C}$ & -1.037110 & -2.696351 & -1.190370 \\
\hline 34 & $\mathrm{C}$ & -2.110902 & -3.059724 & -2.005491 \\
\hline 35 & $\mathrm{C}$ & -6.937687 & -1.885514 & -3.018588 \\
\hline 36 & $\mathrm{C}$ & -7.772550 & -0.811478 & -3.364500 \\
\hline 37 & $\mathrm{C}$ & -7.247696 & 0.449532 & -3.674064 \\
\hline 38 & $\mathrm{C}$ & -5.849874 & 0.597383 & -3.619131 \\
\hline 39 & $\mathrm{C}$ & -5.018394 & -0.462881 & -3.270999 \\
\hline 40 & $\mathrm{C}$ & -7.889627 & -8.394010 & -0.908953 \\
\hline 41 & $\mathrm{C}$ & -7.747410 & -9.224159 & -2.037310 \\
\hline 42 & $\mathrm{C}$ & -8.863710 & -9.690647 & -2.730965 \\
\hline 43 & $\mathrm{C}$ & -10.172622 & -9.357746 & -2.333440 \\
\hline 44 & $\mathrm{C}$ & -10.307602 & -8.537031 & -1.206443 \\
\hline 45 & $\mathrm{C}$ & -9.189291 & -8.060307 & -0.509855 \\
\hline 46 & $\mathrm{H}$ & -3.711223 & -5.061093 & -6.145378 \\
\hline 47 & $\mathrm{C}$ & 0.022713 & -1.343815 & 0.663330 \\
\hline 48 & $\mathrm{C}$ & -0.214223 & -0.285996 & 1.741509 \\
\hline 49 & $\mathrm{C}$ & 1.061586 & 0.109094 & 2.491555 \\
\hline 50 & $\mathrm{C}$ & 0.827737 & 1.188258 & 3.551874 \\
\hline 51 & $\mathrm{C}$ & 2.099234 & 1.624963 & 4.285023 \\
\hline 52 & $\mathrm{C}$ & 1.848383 & 2.713339 & 5.330104 \\
\hline 53 & $\mathrm{C}$ & -8.087987 & 1.646791 & -4.065724 \\
\hline 54 & $\mathrm{C}$ & -9.609362 & 1.492113 & -4.046878 \\
\hline 55 & $\mathrm{C}$ & -10.315251 & 2.799861 & -4.426022 \\
\hline 56 & $\mathrm{C}$ & -11.843515 & 2.714606 & -4.427144 \\
\hline 57 & $\mathrm{C}$ & -12.521928 & 4.042647 & -4.782619 \\
\hline 58 & $\mathrm{C}$ & -2.171794 & -11.578954 & 0.531770 \\
\hline 59 & $\mathrm{C}$ & -2.435361 & -13.084996 & 0.579276 \\
\hline 60 & $\mathrm{C}$ & -1.126881 & -13.880625 & 0.649144 \\
\hline 61 & $\mathrm{C}$ & -1.301866 & -15.401367 & 0.631198 \\
\hline 62 & $\mathrm{C}$ & 0.021240 & -16.159169 & 0.787961 \\
\hline 63 & $\mathrm{C}$ & -0.129161 & -17.677610 & 0.682315 \\
\hline 64 & $\mathrm{C}$ & -11.358084 & -9.876785 & -3.125223 \\
\hline 65 & $\mathrm{C}$ & -12.749243 & -9.560273 & -2.568963 \\
\hline 66 & $\mathrm{C}$ & -13.883755 & -10.087471 & -3.454719 \\
\hline 67 & $\mathrm{C}$ & -15.279064 & -9.793615 & -2.892148 \\
\hline 68 & $\mathrm{C}$ & -16.425064 & -10.269028 & -3.791499 \\
\hline 69 & $\mathrm{C}$ & -17.811569 & -9.980406 & -3.210966 \\
\hline
\end{tabular}




\begin{tabular}{|c|c|c|c|c|}
\hline 70 & $\mathrm{C}$ & -14.049481 & 3.961074 & -4.788023 \\
\hline 71 & $\mathrm{H}$ & -7.777362 & 1.963991 & -5.077589 \\
\hline 72 & $\mathrm{H}$ & -7.810895 & 2.488018 & -3.405487 \\
\hline 73 & $\mathrm{H}$ & -9.942034 & 1.173956 & -3.043286 \\
\hline 74 & $\mathrm{H}$ & -9.917532 & 0.695233 & -4.745552 \\
\hline 75 & $\mathrm{H}$ & -9.970204 & 3.121995 & -5.425196 \\
\hline 76 & $\mathrm{H}$ & -9.997655 & 3.593951 & -3.726185 \\
\hline 77 & $\mathrm{H}$ & -12.193358 & 2.381912 & -3.432988 \\
\hline 78 & $\mathrm{H}$ & -12.169595 & 1.937153 & -5.141279 \\
\hline 79 & $\mathrm{H}$ & -12.166939 & 4.376237 & -5.773542 \\
\hline 80 & $\mathrm{H}$ & -12.194002 & 4.816393 & -4.066623 \\
\hline 81 & $\mathrm{H}$ & -14.434450 & 3.654650 & -3.802460 \\
\hline 82 & $\mathrm{H}$ & -14.404364 & 3.223969 & -5.525282 \\
\hline 83 & $\mathrm{H}$ & -14.505812 & 4.930426 & -5.039047 \\
\hline 84 & $\mathrm{H}$ & -5.682410 & -7.363797 & -2.958455 \\
\hline 85 & $\mathrm{H}$ & -5.752627 & -3.264621 & -0.020186 \\
\hline 86 & $\mathrm{H}$ & -3.304363 & -1.817903 & -4.814380 \\
\hline 87 & $\mathrm{H}$ & -7.733745 & -8.693647 & 2.548954 \\
\hline 88 & $\mathrm{H}$ & -3.931550 & -7.322289 & -0.142881 \\
\hline 89 & $\mathrm{H}$ & -2.095163 & -8.910929 & 0.206578 \\
\hline 90 & $\mathrm{H}$ & -4.933628 & -12.132197 & 0.554347 \\
\hline 91 & $\mathrm{H}$ & -6.777244 & -10.542844 & 0.214592 \\
\hline 92 & $\mathrm{H}$ & -4.504154 & -1.087013 & -0.622362 \\
\hline 93 & $\mathrm{H}$ & -2.622224 & -0.465670 & 0.815654 \\
\hline 94 & $\mathrm{H}$ & -0.059265 & -3.151564 & -1.366523 \\
\hline 95 & $\mathrm{H}$ & -1.946432 & -3.787226 & -2.801052 \\
\hline 96 & $\mathrm{H}$ & -7.387977 & -2.854325 & -2.794402 \\
\hline 97 & $\mathrm{H}$ & -8.850825 & -0.974899 & -3.390652 \\
\hline 98 & $\mathrm{H}$ & -5.404386 & 1.567544 & -3.853924 \\
\hline 99 & $\mathrm{H}$ & -3.941458 & -0.297243 & -3.225670 \\
\hline 100 & $\mathrm{H}$ & -6.751396 & -9.522256 & -2.368999 \\
\hline 101 & $\mathrm{H}$ & -9.344635 & -7.414100 & 0.355499 \\
\hline 102 & $\mathrm{H}$ & -8.714848 & -10.328390 & -3.606442 \\
\hline 103 & $\mathrm{H}$ & -11.300659 & -8.251404 & -0.856824 \\
\hline 104 & $\mathrm{H}$ & -1.604137 & -11.287753 & 1.434547 \\
\hline 105 & $\mathrm{H}$ & -1.489576 & -11.372458 & -0.312063 \\
\hline 106 & $\mathrm{H}$ & -3.005653 & -13.391345 & -0.315118 \\
\hline 107 & $\mathrm{H}$ & -3.063606 & -13.335331 & 1.451608 \\
\hline 108 & $\mathrm{H}$ & -0.573923 & -13.589645 & 1.561029 \\
\hline 109 & $\mathrm{H}$ & -0.482928 & -13.586521 & -0.199366 \\
\hline 110 & $\mathrm{H}$ & -1.784933 & -15.702321 & -0.315946 \\
\hline 111 & $\mathrm{H}$ & -1.994711 & -15.707336 & 1.435662 \\
\hline 112 & $\mathrm{H}$ & 0.471450 & -15.898213 & 1.762024 \\
\hline 113 & $\mathrm{H}$ & 0.734677 & -15.806042 & 0.022890 \\
\hline 114 & $\mathrm{H}$ & 0.831653 & -18.191165 & 0.835950 \\
\hline 115 & $\mathrm{H}$ & -0.508924 & -17.972584 & -0.308758 \\
\hline 116 & $\mathrm{H}$ & -0.836853 & -18.060628 & 1.434493 \\
\hline 117 & $\mathrm{H}$ & 0.801240 & -0.978515 & -0.030544 \\
\hline 118 & $\mathrm{H}$ & 0.454418 & -2.247649 & 1.128990 \\
\hline 119 & $\mathrm{H}$ & -0.960969 & -0.652409 & 2.467443 \\
\hline 120 & $\mathrm{H}$ & -0.651108 & 0.616945 & 1.280961 \\
\hline
\end{tabular}




$\begin{array}{lllll}121 & \mathrm{H} & 1.812840 & 0.467690 & 1.765015 \\ 122 & \mathrm{H} & 1.503907 & -0.784534 & 2.967459 \\ 123 & \mathrm{H} & 0.089505 & 0.824257 & 4.289281 \\ 124 & \mathrm{H} & 2.364672 & 2.070792 & 3.074249 \\ 125 & \mathrm{H} & 2.835975 & 0.984579 & 3.545775 \\ 126 & \mathrm{H} & 1.156699 & 2.362900 & 4.768203 \\ 127 & \mathrm{H} & 1.397111 & 3.606461 & 6.112406 \\ 128 & \mathrm{H} & 2.781637 & 3.025609 & 4.869775 \\ 129 & \mathrm{H} & -11.254798 & -10.971655 & 5.821812 \\ 130 & \mathrm{H} & -11.285891 & -9.481616 & -3.228092 \\ 131 & \mathrm{H} & -12.865506 & -8.468412 & -4.154968 \\ 132 & \mathrm{H} & -12.848529 & -9.987607 & -2.457919 \\ 133 & \mathrm{H} & -13.768090 & -11.178044 & -1.555967 \\ 134 & \mathrm{H} & -13.794449 & -9.643322 & -3.590453 \\ 135 & \mathrm{H} & -15.381164 & -8.706069 & -2.462304 \\ 136 & \mathrm{H} & -15.377953 & -10.266105 & -1.898227 \\ 137 & \mathrm{H} & -16.319674 & -11.353541 & -3.970503 \\ 138 & \mathrm{H} & -16.331740 & -9.787889 & -4.780379 \\ 139 & \mathrm{H} & -17.946867 & -10.475737 & -2.236974 \\ 140 & \mathrm{H} & -18.609562 & -10.334892 & -3.880333 \\ 141 & \mathrm{H} & -17.961314 & -8.899795 & -3.057031 \\ 142 & \mathrm{H} & & & \end{array}$

Table S4. The Cartesian coordinates $(\AA)$ of the optimized geometries for ISOAA-H in excited state.

\begin{tabular}{lcccc}
\hline & ATOM & $\mathrm{X}$ & $\mathrm{Y}$ & $\mathrm{Z}$ \\
\hline 1 & $\mathrm{C}$ & -6.210845 & -5.385217 & -0.188345 \\
2 & $\mathrm{C}$ & -6.179267 & -6.557268 & -1.010171 \\
3 & $\mathrm{C}$ & -5.723163 & -6.495052 & -2.312275 \\
4 & $\mathrm{C}$ & -5.242186 & -5.253246 & -2.806899 \\
5 & $\mathrm{C}$ & -5.261432 & -4.094208 & -1.973866 \\
6 & $\mathrm{C}$ & -5.752350 & -4.141539 & -0.674759 \\
7 & $\mathrm{C}$ & -4.595809 & -2.908762 & -2.682469 \\
8 & $\mathrm{C}$ & -4.197022 & -3.545780 & -4.028917 \\
9 & $\mathrm{C}$ & -4.619386 & -4.943151 & -4.078534 \\
10 & $\mathrm{O}$ & -4.409740 & -5.717813 & -5.096569 \\
11 & $\mathrm{O}$ & -3.218023 & -4.479000 & -6.789690 \\
12 & $\mathrm{C}$ & -3.074206 & -3.288418 & -6.311216 \\
13 & $\mathrm{C}$ & -3.542851 & -2.835783 & -4.989530 \\
14 & $\mathrm{C}$ & -6.705630 & -5.792556 & 1.097683 \\
15 & $\mathrm{C}$ & -6.979298 & -7.152723 & -0.2135341 \\
16 & $\mathrm{C}$ & -6.653136 & -7.797559 & 2.363802 \\
17 & $\mathrm{C}$ & -7.474817 & -7.652089 & 3.322229 \\
18 & $\mathrm{C}$ & -7.621949 & -6.675695 & 2.731604 \\
19 & $\mathrm{Se}$ & -7.089901 & -4.945824 & -0.010782 \\
20 & $\mathrm{C}$ & -5.504678 & -8.810187 & 0.011189 \\
21 & $\mathrm{C}$ & -4.167849 & -8.376377 & 0.209807 \\
22 & $\mathrm{C}$ & -3.126620 & -9.278727 & 0.391704 \\
23 & $\mathrm{C}$ & -3.361856 & -10.652361 & 0.406625 \\
24 & $\mathrm{C}$ & -4.696466 & -11.076912 & 0.216371 \\
25 & $\mathrm{C}$ & -5.749702 & -10.170177 & 5.072558 \\
26 & $\mathrm{Br}$ & -8.311729 & -6.917911 &
\end{tabular}




\begin{tabular}{|c|c|c|c|c|}
\hline 27 & $\mathrm{O}$ & -2.462580 & -2.445061 & -7.048756 \\
\hline 28 & $\mathrm{C}$ & -3.378772 & -2.482736 & -1.836111 \\
\hline 29 & $\mathrm{C}$ & -5.545235 & -1.731969 & -2.971033 \\
\hline 30 & $\mathrm{C}$ & -3.534099 & -1.558905 & -0.791969 \\
\hline 31 & $\mathrm{C}$ & -2.457322 & -1.199366 & 0.023973 \\
\hline 32 & $\mathrm{C}$ & -1.182184 & -1.748218 & -0.163456 \\
\hline 33 & $\mathrm{C}$ & -1.037685 & -2.695232 & -1.191519 \\
\hline 34 & $\mathrm{C}$ & -2.110110 & -3.057660 & -2.008352 \\
\hline 35 & $\mathrm{C}$ & -6.935606 & -1.886159 & -3.023031 \\
\hline 36 & $\mathrm{C}$ & -7.770581 & -0.811654 & -3.364874 \\
\hline 37 & $\mathrm{C}$ & -7.247619 & 0.450314 & -3.674139 \\
\hline 38 & $\mathrm{C}$ & -5.850024 & 0.598537 & -3.619685 \\
\hline 39 & $\mathrm{C}$ & -5.018814 & -0.462393 & -3.275015 \\
\hline 40 & $\mathrm{C}$ & -7.888896 & -8.394191 & -0.909654 \\
\hline 41 & $\mathrm{C}$ & -7.748780 & -9.224735 & -2.037110 \\
\hline 42 & $\mathrm{C}$ & -8.865460 & -9.690144 & -2.729895 \\
\hline 43 & $\mathrm{C}$ & -10.173867 & -9.357298 & -2.332672 \\
\hline 44 & $\mathrm{C}$ & -10.307695 & -8.536448 & -1.206286 \\
\hline 45 & $\mathrm{C}$ & -9.188579 & -8.061139 & -0.511131 \\
\hline 46 & $\mathrm{H}$ & -3.781160 & -5.121219 & -6.035706 \\
\hline 47 & $\mathrm{C}$ & 0.021657 & -1.344085 & 0.663527 \\
\hline 48 & $\mathrm{C}$ & -0.213820 & -0.286296 & 1.741419 \\
\hline 49 & $\mathrm{C}$ & 1.061220 & 0.109399 & 2.491384 \\
\hline 50 & $\mathrm{C}$ & 0.828148 & 1.187846 & 3.551585 \\
\hline 51 & $\mathrm{C}$ & 2.098755 & 1.624969 & 4.284602 \\
\hline 52 & $\mathrm{C}$ & 1.848290 & 2.712549 & 5.329527 \\
\hline 53 & $\mathrm{C}$ & -8.088337 & 1.646445 & -4.065453 \\
\hline 54 & $\mathrm{C}$ & -9.609122 & 1.492580 & -4.046866 \\
\hline 55 & $\mathrm{C}$ & -10.315556 & 2.799335 & -4.425627 \\
\hline 56 & $\mathrm{C}$ & -11.843179 & 2.714531 & -4.427115 \\
\hline 57 & $\mathrm{C}$ & -12.522069 & 4.041634 & -4.782019 \\
\hline 58 & $\mathrm{C}$ & -2.172309 & -11.578990 & 0.532153 \\
\hline 59 & $\mathrm{C}$ & -2.434792 & -13.084639 & 0.578680 \\
\hline 60 & $\mathrm{C}$ & -1.127592 & -13.880955 & 0.649455 \\
\hline 61 & $\mathrm{C}$ & -1.301826 & -15.401007 & 0.630913 \\
\hline 62 & $\mathrm{C}$ & 0.020208 & -16.159227 & 0.787185 \\
\hline 63 & $\mathrm{C}$ & -0.129808 & -17.677106 & 0.682533 \\
\hline 64 & $\mathrm{C}$ & -11.357818 & -9.876421 & -3.124598 \\
\hline 65 & $\mathrm{C}$ & -12.748691 & -9.560634 & -2.569660 \\
\hline 66 & $\mathrm{C}$ & -13.883626 & -10.087340 & -3.454176 \\
\hline 67 & $\mathrm{C}$ & -15.278237 & -9.793691 & -2.892477 \\
\hline 68 & $\mathrm{C}$ & -16.423321 & -10.269166 & -3.791227 \\
\hline 69 & $\mathrm{C}$ & -17.809354 & -9.980911 & -3.211333 \\
\hline 70 & $\mathrm{C}$ & -14.048923 & 3.960102 & -4.787727 \\
\hline 71 & $\mathrm{H}$ & -7.778199 & 1.963873 & -5.077207 \\
\hline 72 & $\mathrm{H}$ & -7.811133 & 2.487933 & -3.406179 \\
\hline 73 & $\mathrm{H}$ & -9.941468 & 1.174269 & -3.043761 \\
\hline 74 & $\mathrm{H}$ & -9.917403 & 0.695712 & -4.744875 \\
\hline 75 & $\mathrm{H}$ & -9.970730 & 3.121618 & -5.424454 \\
\hline 76 & $\mathrm{H}$ & -9.998295 & 3.593338 & -3.726129 \\
\hline 77 & $\mathrm{H}$ & -12.193102 & 2.381740 & -3.433519 \\
\hline
\end{tabular}




\begin{tabular}{|c|c|c|c|c|}
\hline 78 & $\mathrm{H}$ & -12.169318 & 1.937452 & -5.141036 \\
\hline 79 & $\mathrm{H}$ & -12.167521 & 4.375657 & -5.772515 \\
\hline 80 & $\mathrm{H}$ & -12.194776 & 4.815236 & -4.066164 \\
\hline 81 & $\mathrm{H}$ & -14.434148 & 3.653702 & -3.802764 \\
\hline 82 & $\mathrm{H}$ & -14.404067 & 3.223529 & -5.524827 \\
\hline 83 & $\mathrm{H}$ & -14.505135 & 4.929108 & -5.038303 \\
\hline 84 & $\mathrm{H}$ & -5.684617 & -7.371270 & -2.956677 \\
\hline 85 & $\mathrm{H}$ & -5.749514 & -3.262009 & -0.024497 \\
\hline 86 & $\mathrm{H}$ & -3.302192 & -1.792788 & -4.801822 \\
\hline 87 & $\mathrm{H}$ & -7.732938 & -8.693526 & 2.548021 \\
\hline 88 & $\mathrm{H}$ & -3.933680 & -7.323206 & -0.143987 \\
\hline 89 & $\mathrm{H}$ & -2.096618 & -8.912051 & 0.204942 \\
\hline 90 & $\mathrm{H}$ & -4.933462 & -12.131218 & 0.555128 \\
\hline 91 & $\mathrm{H}$ & -6.775728 & -10.541752 & 0.215843 \\
\hline 92 & $\mathrm{H}$ & -4.503123 & -1.088453 & -0.623128 \\
\hline 93 & $\mathrm{H}$ & -2.623260 & -0.467484 & 0.814592 \\
\hline 94 & $\mathrm{H}$ & -0.060486 & -3.151505 & -1.364993 \\
\hline 95 & $\mathrm{H}$ & -1.948148 & -3.789389 & -2.799850 \\
\hline 96 & $\mathrm{H}$ & -7.383850 & -2.854129 & -2.794544 \\
\hline 97 & $\mathrm{H}$ & -8.848516 & -0.974739 & -3.390295 \\
\hline 98 & $\mathrm{H}$ & -5.405430 & 1.569464 & -3.851667 \\
\hline 99 & $\mathrm{H}$ & -3.942853 & -0.295810 & -3.222058 \\
\hline 100 & $\mathrm{H}$ & -6.752838 & -9.521854 & -2.369029 \\
\hline 101 & $\mathrm{H}$ & -9.341949 & -7.415550 & 0.353719 \\
\hline 102 & $\mathrm{H}$ & -8.716906 & -10.328713 & -3.604288 \\
\hline 103 & $\mathrm{H}$ & -11.299899 & -8.249813 & -0.855935 \\
\hline 104 & $\mathrm{H}$ & -1.606346 & -11.289379 & 1.436093 \\
\hline 105 & $\mathrm{H}$ & -1.488188 & -11.372176 & -0.309658 \\
\hline 106 & $\mathrm{H}$ & -3.005256 & -13.390890 & -0.315103 \\
\hline 107 & $\mathrm{H}$ & -3.063092 & -13.334583 & 1.450475 \\
\hline 108 & $\mathrm{H}$ & -0.576312 & -13.590533 & 1.562056 \\
\hline 109 & $\mathrm{H}$ & -0.482209 & -13.586450 & -0.197318 \\
\hline 110 & $\mathrm{H}$ & -1.785051 & -15.701965 & -0.315696 \\
\hline 111 & $\mathrm{H}$ & -1.993971 & -15.706775 & 1.435507 \\
\hline 112 & $\mathrm{H}$ & 0.470473 & -15.898344 & 1.760697 \\
\hline 113 & $\mathrm{H}$ & 0.733686 & -15.806791 & 0.022333 \\
\hline 114 & $\mathrm{H}$ & 0.830700 & -18.190495 & 0.836111 \\
\hline 115 & $\mathrm{H}$ & -0.509855 & -17.972769 & -0.307791 \\
\hline 116 & $\mathrm{H}$ & -0.837197 & -18.059641 & 1.434703 \\
\hline 117 & $\mathrm{H}$ & 0.801179 & -0.979172 & -0.029076 \\
\hline 118 & $\mathrm{H}$ & 0.452862 & -2.247548 & 1.129539 \\
\hline 119 & $\mathrm{H}$ & -0.960307 & -0.652305 & 2.467158 \\
\hline 120 & $\mathrm{H}$ & -0.650681 & 0.616514 & 1.281436 \\
\hline 121 & $\mathrm{H}$ & 1.812356 & 0.468417 & 1.765453 \\
\hline 122 & $\mathrm{H}$ & 1.503660 & -0.783804 & 2.966919 \\
\hline 123 & $\mathrm{H}$ & 0.090307 & 0.823962 & 4.288797 \\
\hline 124 & $\mathrm{H}$ & 0.365283 & 2.070364 & 3.074629 \\
\hline 125 & $\mathrm{H}$ & 2.835203 & 1.985058 & 3.545802 \\
\hline 126 & $\mathrm{H}$ & 2.561217 & 0.747083 & 4.767645 \\
\hline 127 & $\mathrm{H}$ & 1.156459 & 2.362749 & 6.111410 \\
\hline 128 & $\mathrm{H}$ & 1.398362 & 3.606110 & 4.869747 \\
\hline
\end{tabular}




\begin{tabular}{llccc}
129 & $\mathrm{H}$ & 2.780965 & 3.023925 & 5.821855 \\
130 & $\mathrm{H}$ & -11.255019 & -10.970961 & -3.229094 \\
131 & $\mathrm{H}$ & -11.286458 & -9.481015 & -4.153997 \\
132 & $\mathrm{H}$ & -12.864660 & -8.468922 & -2.458829 \\
133 & $\mathrm{H}$ & -12.848175 & -9.986630 & -1.556355 \\
134 & $\mathrm{H}$ & -13.769021 & -11.177676 & -3.590662 \\
135 & $\mathrm{H}$ & -13.795104 & -9.643794 & -4.461835 \\
136 & $\mathrm{H}$ & -15.380590 & -8.706505 & -2.722697 \\
137 & $\mathrm{H}$ & -15.377840 & -10.265339 & -1.898659 \\
138 & $\mathrm{H}$ & -16.318493 & -11.353582 & -3.969960 \\
139 & $\mathrm{H}$ & -16.330546 & -9.788277 & -4.780023 \\
140 & $\mathrm{H}$ & -17.946280 & -10.476341 & -2.237908 \\
141 & $\mathrm{H}$ & -18.607569 & -10.334117 & -3.880557 \\
142 & $\mathrm{H}$ & -17.959884 & -8.900867 & -3.055938 \\
\hline
\end{tabular}

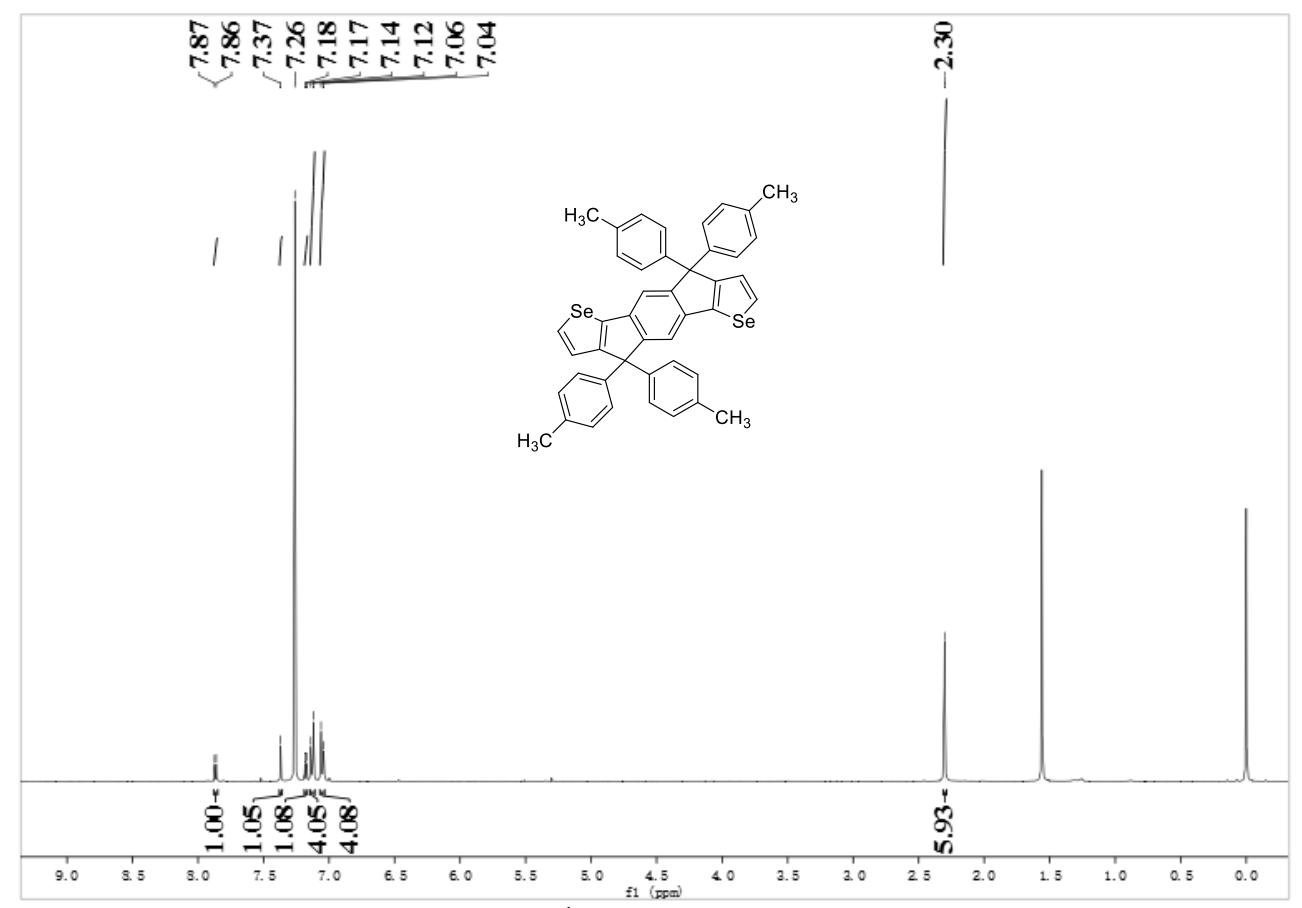

Figure S9. ${ }^{1} \mathrm{H}-\mathrm{NMR}$ of IDS-M. 


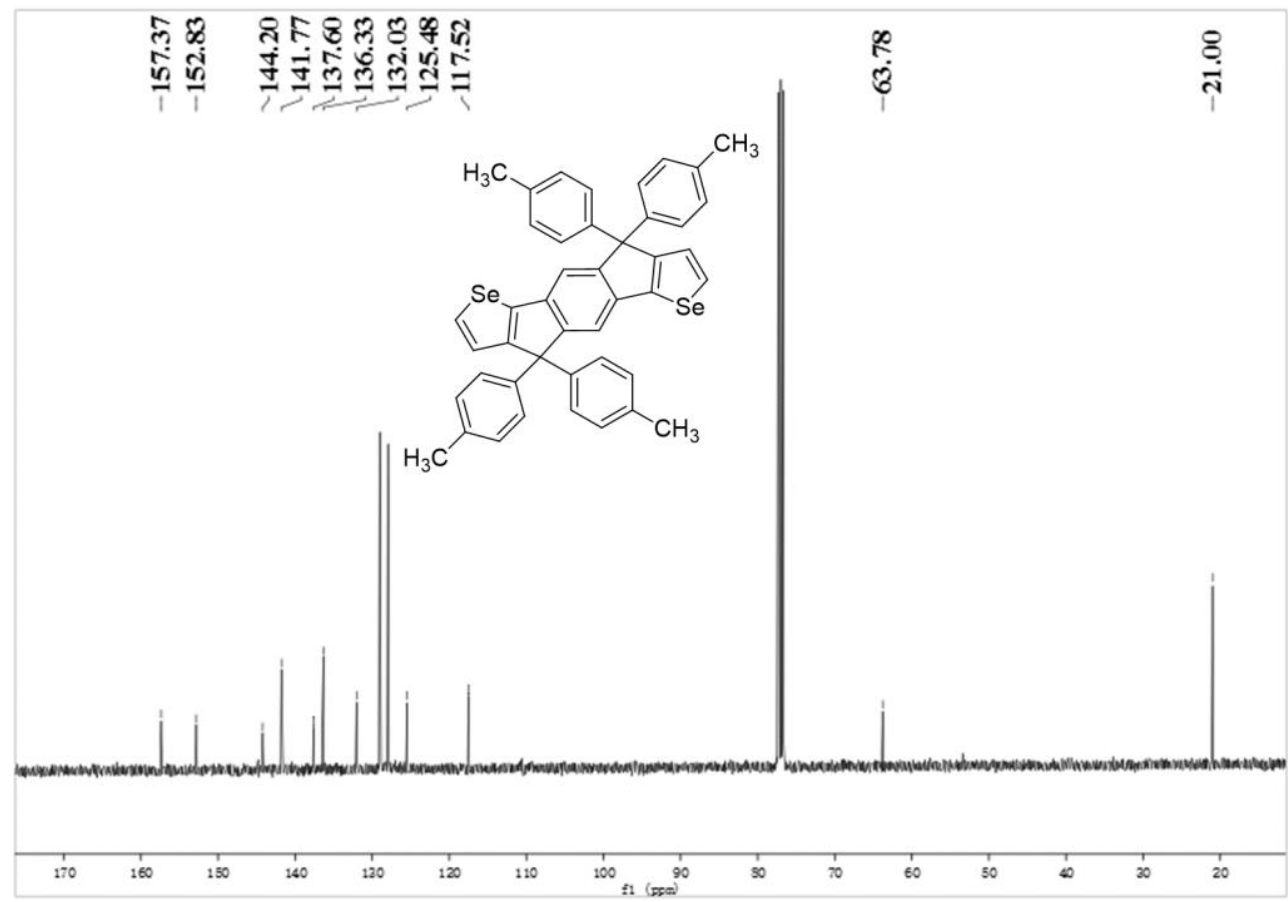

Figure S10. ${ }^{13} \mathrm{C}-\mathrm{NMR}$ of IDS-M

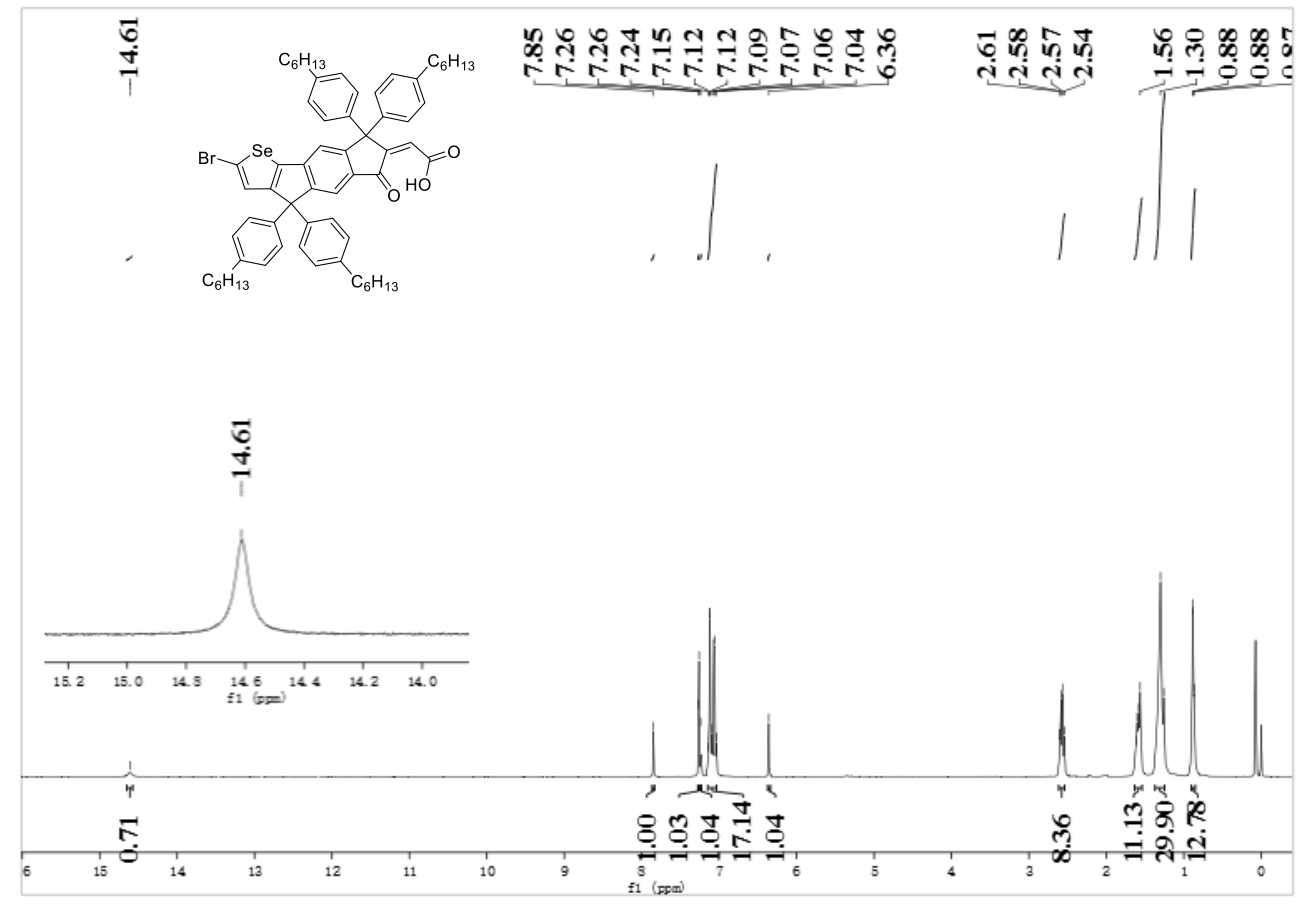

Figure S11. ${ }^{1} \mathrm{H}-\mathrm{NMR}$ of ISOAA-H. 


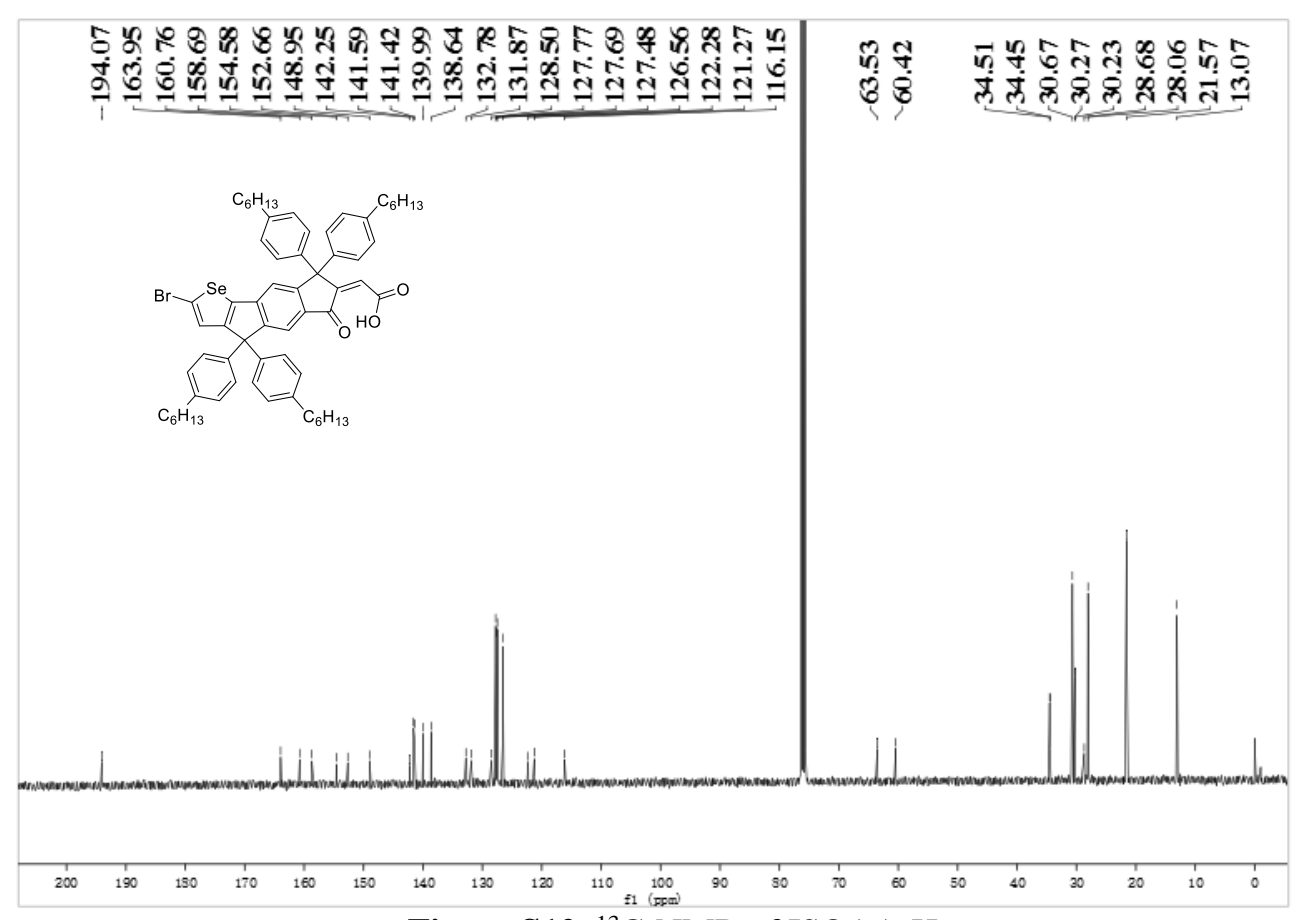

Figure S12. ${ }^{13} \mathrm{C}-\mathrm{NMR}$ of ISOAA-H

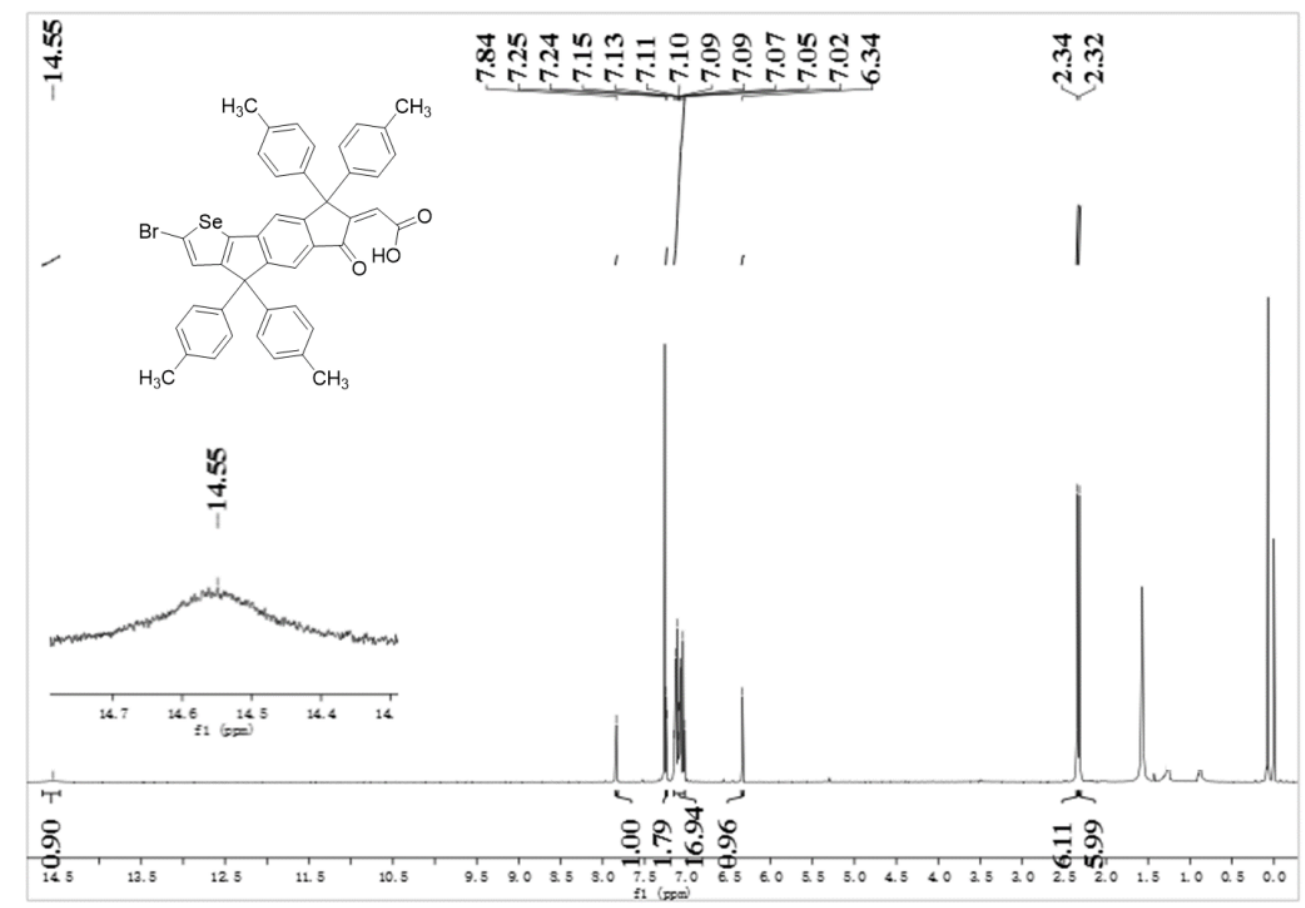

Figure S13. ${ }^{1} \mathrm{H}-\mathrm{NMR}$ of ISOAA-M. 


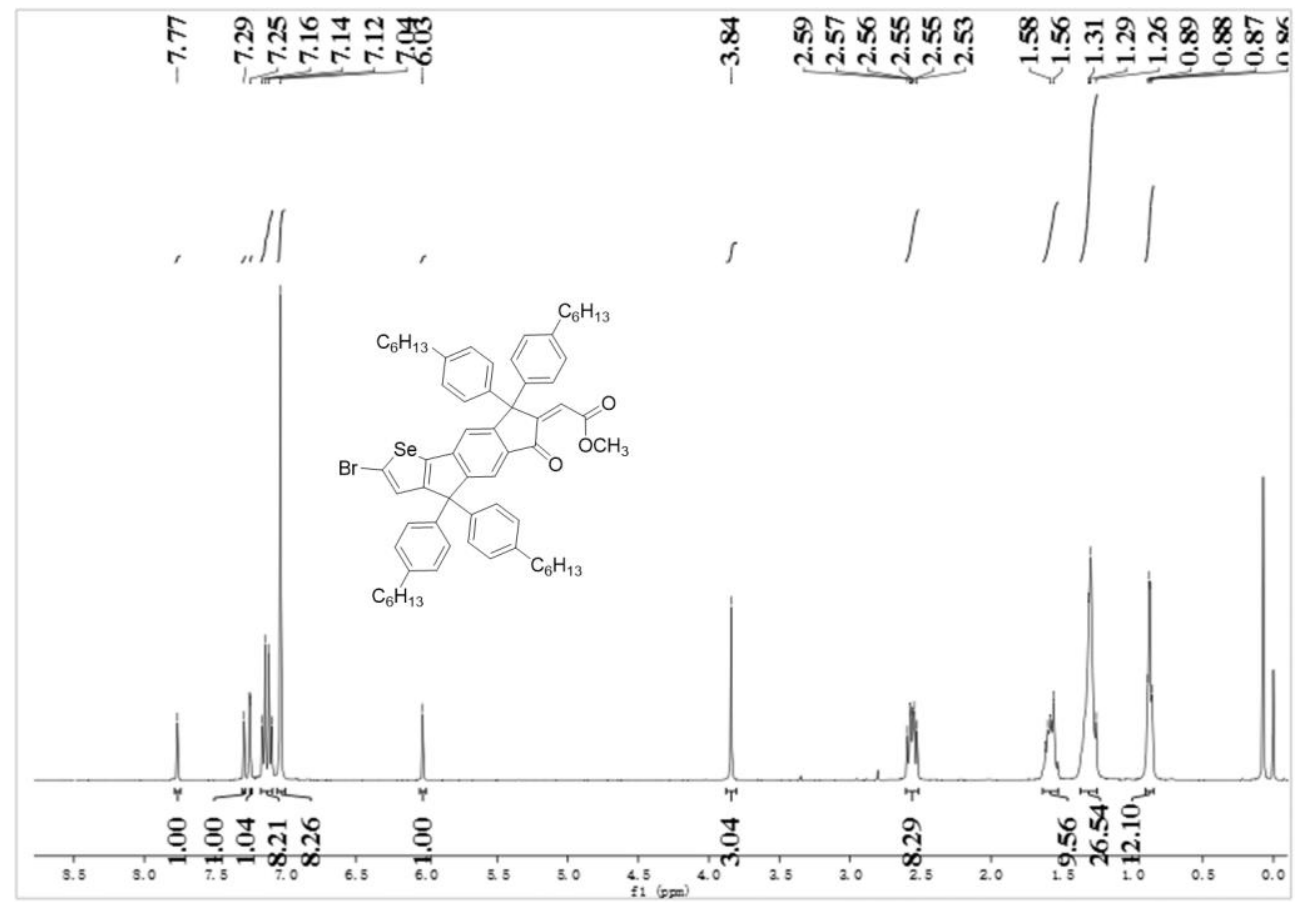

Figure S14. ${ }^{1} \mathrm{H}-\mathrm{NMR}$ of ISOAM-H.

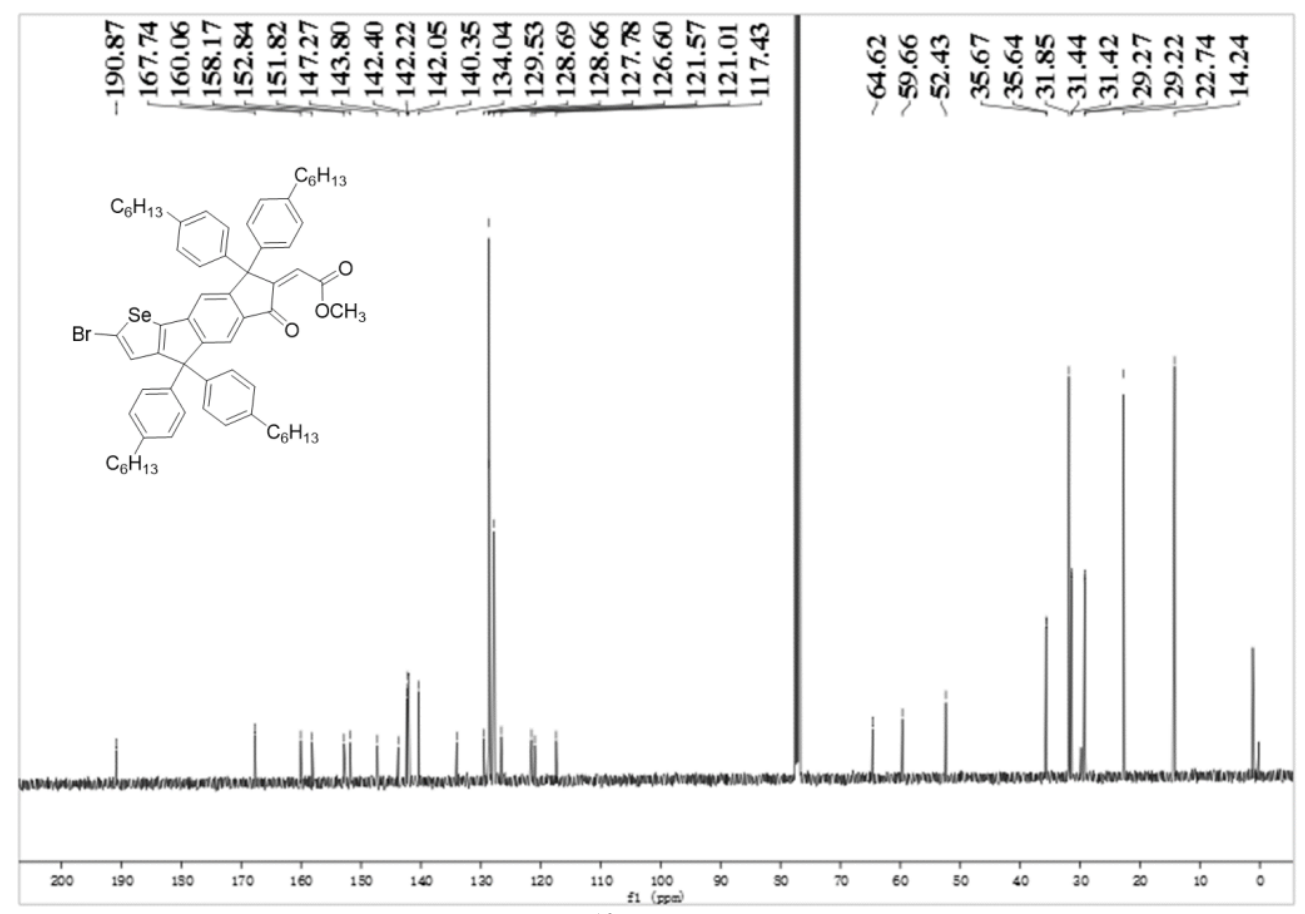

Figure S15. ${ }^{13} \mathrm{C}-\mathrm{NMR}$ of ISOAM-H.

\section{References}

(1) Lakowicz, J. R., Principles of Fluorescence Spectroscopy, 3rd Ed. Springer: 2006.

(2) Lippert, V. E., Spektroskopische Bestimmung Des Dipolmomentes Aromatischer Verbindungen Im Ersten Angeregten Singulettzustand. Z. Elektrochem. 1957, 61, 962-975.

(3) Mataga, N.; Kaifu, Y.; Koizumi, M., Solvent Effects Upon Fluorescence Spectra and the Dipolemoments of Excited Molecules. Bull. Chem. Soc. Jpn. 1956, 29, 465-470. 